VOL. 50 (1994) [399-404]

\title{
INDUCED REPRESENTATIONS TWISTED BY COCYCLES
}

\author{
HuU Hung Bui
}

\begin{abstract}
We define a notion of representation induced from cocycles which encompasses the case of Kawakami's generalised induced representation. Our purpose is to formulate Kawakami's construction within the context of Rieffel's formalism of induced representations of $\mathrm{C}^{*}$-algebras.
\end{abstract}

\section{INTRODUCTION}

Rieffel developed in [5] an elegant method of inducing representations of $C^{*}$ algebras, and showed that Mackey's induced representations can be obtained through this process. On the other hand, Kawakami presented in [3] a notion of induced representation under a very general situation: through a double transformation group $(G, X, H)$ and twisted by a cocycle $\gamma$ of $(G, X, H)$.

Our purpose is to formulate Kawakami's construction within the context of Rieffel's formalism of induced representations of $C^{*}$-algebras. In Section 2, we establish a bijective corresponding between $G$-invariant cocycles on $X \times H$ and cocycles of $(G, X, H)$ extending the result of Kawakami, and then define a notion of representation induced from $G$-invariant cocycles on $X \times H$. In Section 3, we show that Kawakami's generalised induced representations can be also obtained through Rieffel's process.

In this paper $G$ and $H$ will be separable locally compact groups, and $X$ will be a separable locally compact space. We suppose that $G$ (respectively, $H$ ) acts continuously on $X$ by the map $(s, x) \longmapsto s x$ with $s \in G$ and $x \in X$ (respectively, $(x, t) \longmapsto x t$ with $x \in X$ and $t \in H$ ). We denote by $\ell_{G}$ and $\ell_{H}$ the left Haar measures on $G$ and $H$, and by $\Delta_{G}$ and $\Delta_{H}$ the modular functions of $G$ and $H$. All Hilbert spaces are assumed to be separable. We shall denote by $\mathcal{S}$ a unital $C^{*}$-subalgebra of the algebra $\mathcal{B}(\mathcal{H})$ of all bounded operators on a Hilbert space $\mathcal{H}$, and by $\mathcal{S}^{\boldsymbol{u}}$ the set of all unitary elements of $\mathcal{S}$ equipped with the Borel structure generated by the weak operator topology.

Received 12th January, 1994

The author would like to thank his graduate advisers at The University of New South Wales, Professor C. E. Sutherland and Professor I. Raeburn.

Copyright Clearance Centre, Inc. Serial-fee code: 0004-9729/94 \$A2.00+0.00. 


\section{COCYClES AND INDUCED REPRESENTATIONS}

A $\mathcal{S}^{u}$-valued cocycle on $X \times H$ is a Borel function $C: X \times H \rightarrow \mathcal{S}^{u}$ such that

$$
C(x, r t)=C(x, r) C(x r, t), \quad \forall x \in X, \forall r, t \in H .
$$

Two cocycles $C$ and $D$ are said to be ( $G$-invariant) cohomologous if there is a ( $G$ invariant) Borel function $f: X \longrightarrow \mathcal{S}^{u}$ such that

$$
C(x, t)=f(x) D(x, t) f(x t)^{*}, \quad \forall x \in X, \forall t \in H
$$

We denote by $Z^{S}(X ; H)$ the set of all $\mathcal{S}^{u}$-valued cocycles on $X \times H$. The quotient set of $Z^{S}(X ; H)$ by the relation of being cohomologous is denoted by $H^{S}(X ; H)$.

If $(G, X, H)$ is a double transformation group, we define $S^{u}$-valued cocycles of $(G, X, H), Z^{\mathcal{S}}(G ; X ; H)$ and $H^{\mathcal{S}}(G ; X ; H)$ in the same way as in [3, Definition 1.4].

For each $\chi \in Z^{\mathcal{S}}(G ; X ; H)$, we put

$$
\tilde{\chi}(x, t)=\chi(x)^{*} \chi(x t), \quad \forall x \in X, \forall t \in H .
$$

Then $\tilde{\chi}$ is a $G$-invariant cocycle on $X \times H$. If $\chi$ and $\gamma$ are cohomologous, then so are $\tilde{\chi}$ and $\tilde{\gamma}$.

We say that $(X, H)$ is smooth if each orbit is locally closed in $X$, and say that $(X, H)$ is effective if each stability group is trivial.

The following result is a generalisation of [3, Proposition 1.5].

Theorem 2.1. Let $(G, X, H)$ be a double transformation group. Suppose that $(X, H)$ is smooth and effective. Then for each $G$-invariant $\mathcal{S}^{u}$-valued cocycle $C$ on $X \times H$, there is a $\chi \in Z^{S}(G ; X ; H)$ such that $C=\tilde{\chi}$. Furthermore, if $(G, X)$ is smooth then there is a bijective corresponding between $H^{\mathcal{S}}(G ; X ; H)$ and $H^{\mathcal{S}}(G \backslash X ; H)$.

Proof: Since $(X, H)$ is smooth, the quotient map $q: X \longrightarrow X / H$ has a Borel cross section $d: X / H \longrightarrow X$. For each $x \in X$, we have $x=d(q(x)) b(x)$ for some $b(x) \in H$. The map $\phi: X / H \times H \longrightarrow X$, defined by $\phi(q(x), t)=d(q(x)) t$, is Borel. Since $(X, H)$ is effective, $\phi$ is surjective. Observe that $\phi^{-1}(x)=(q(x), b(x))$. Since $X / H \times H$ is a standard space, $\phi^{-1}$ is Borel (see [4, p.72]), and hence $b$ is Borel. If $C$ is a $G$-invariant $S^{u}$-valued cocycle on $X \times H$ and if we put $\chi(x)=C(d(q(x)), b(x))$, then $\chi \in Z^{\mathcal{S}}(G ; X ; H)$.

Now if $(G, X)$ is smooth, the quotient map $p: X \rightarrow G \backslash X$ has a Borel cross section $c: G \backslash X \longrightarrow X$. If $\chi \in Z^{\mathcal{S}}(G ; X ; H)$ and if we put

$$
\widehat{\chi}(p(x), t)=\tilde{\chi}(c(p(x)), t)=\tilde{\chi}(x, t),
$$


then $\widehat{\chi}: G \backslash X \times H \longrightarrow \mathcal{S}^{u}$ is Borel. If $\chi$ and $\gamma$ are cohomologous, then so are $\hat{\chi}$ and $\hat{\gamma}$. Therefore the map $[\chi] \longmapsto[\hat{\chi}]$ from $H^{\mathcal{S}}(G ; X ; H)$ into $H^{\mathcal{S}}(G \backslash X ; H)$ is well-defined. By the first part this map is surjective. Suppose that $\widehat{\chi}$ and $\widehat{\gamma}$ are cohomologous, that is, there is a Borel map $f: G \backslash X \longrightarrow \mathcal{S}^{u}$ such that

$$
\widehat{\chi}(p(x), t)=f(p(x)) \hat{\gamma}(p(x), t) f(p(x) t)^{*}, \quad \forall x \in X, \forall t \in H .
$$

If we put $N(x)=f(p(x))^{*}$ and $M(x)=\chi(x) N(x)^{*} \gamma(x)^{*}$, then $\chi(x)=M(x) \gamma(x) N(x)$. Therefore this map is also injective.

From now on we suppose that there is a positive measure $\mu$ on $X / H$ such that for each $s \in G$, the measure $\mu_{s}$, defined by

$$
\mu_{s}(F)=\int_{X / H} F\left(s^{-1} \dot{x}\right) d \mu(\dot{x}), \quad \forall F \in C_{c}(X / H),
$$

is equivalent to $\mu$. We also suppose that $H$ acts properly on $X$. By [1, Chapter VII Section 2, Proposition 4], there is a positive measure $\nu$ on $X$ such that

$$
\nu(f)=\int_{X / H} d \mu(\dot{x}) \int_{H} f(x t) d \ell_{H}(t), \quad \forall f \in C_{c}(X) .
$$

We deduce from [6, Theorem 5.10] that there is a $H$-invariant $\ell_{G} \otimes \nu$-measurable function $\rho: G \times X \longrightarrow(0, \infty)$ such that for each $s \in G$, the function $x \longmapsto \rho(s, x)$ is a Radon-Nikodym derivative of $\nu_{s}$ with respect to $\nu$.

Let $C$ be a $G$-invariant $\mathcal{S}^{u}$-valued cocycle on $X \times H$. We denote by $\mathcal{H}^{C}$ the set of (equivalence classes of) all $\nu$-measurable maps $\phi: X \longrightarrow \mathcal{H}$ such that for $\nu$-almost all $x \in X$ we have $\phi(x t)=C(x, t)^{*} \phi(x)$ for all $t \in H$, and

$$
\int_{X / H}\langle\phi(x) \mid \phi(x)\rangle d \mu(\dot{x})<\infty .
$$

Then $\mathcal{H}^{C}$ is a separable Hilbert space with the inner product given by

$$
\langle\phi \mid \psi\rangle=\int_{X / H}\langle\phi(x) \mid \psi(x)\rangle d \mu(\dot{x})
$$

For each $s \in G$ we define a unitary operator $U_{s}^{C}$ on $\mathcal{H}^{C}$ by

$$
\left(U_{s}^{C} \phi\right)(x)=\rho\left(s^{-1}, x\right)^{1 / 2} \phi\left(s^{-1} x\right), \quad \forall \phi \in \mathcal{H}^{C} .
$$

PROPOSITION 2.2. $\left(U^{C}, \mathcal{H}^{C}\right)$ is a continuous unitary representation of $G$. If $C$ and $D$ are $G$-invariant cohomologous then $\left(U^{C}, \mathcal{H}^{C}\right)$ and $\left(U^{D}, \mathcal{H}^{D}\right)$ are unitarily equivalent.

Proof: The first assertion follows from routine arguments. Let $f: X \longrightarrow \mathcal{S}^{u}$ be a $G$-invariant Borel map satisfying (2.1). We define

$$
(T \phi)(x)=f(x)^{*} \phi(x), \quad \forall \phi \in \mathcal{H}^{C} .
$$

Then $T$ defines a unitary intertwining operator between $\left(U^{C}, \mathcal{H}^{C}\right)$ and $\left(U^{D}, \mathcal{H}^{D}\right)$. 
REMARK 2.3. Suppose that $(L, \mathcal{H})$ is a continuous unitary representation of $H$. Put $\mathcal{M}=\left\{L_{t}: t \in H\right\}^{\prime}$. Pick $\gamma \in Z^{\mathcal{M}}(G ; X ; H)$, and put

$$
C(x, t)=\gamma(x)^{*} \gamma(x t) L_{t}, \quad \forall x \in X, \forall t \in H .
$$

Then $C$ is a $G$-invariant $\mathcal{M}^{u}$-valued cocycle, and $\left(U^{C}, \mathcal{H}^{C}\right)$ is just Kawakami's generalised induced representation $\left(U^{\gamma}, \mathcal{H}^{\gamma}\right)$ from $L$ through $(G, X, H)$ twisted by $\gamma$ as defined in $[3$, p.676].

\section{INDUCING CONSTRUCTION WITHIN RIEFFEL'S FRAMEWORK}

In this section we bring Kawakami's construction of generalised induced representations into Rieffel's framework of induced representations of $C^{*}$-algebras.

We denote by $\iota$ the trival action of $H$ on $\mathcal{S}$. Put $\mathcal{B}=C^{*}(\mathcal{S}, H, \iota), \mathcal{B}_{c}=C_{c}(H, \mathcal{S})$, $\mathcal{A}=C^{*}(G)$ and $\mathcal{A}_{\mathrm{c}}=C_{c}(G)$. Let $\mathcal{V}$ denote the subspace of $L^{2}(X, \nu ; \mathcal{S})$ consisting of maps vanishing outside a compact subset of $X$.

Let $C$ be a $G$-invariant $\mathcal{S}^{u}$-valued cocycle on $X \times H$. By Theorem 2.1 there is a cocycle $\chi$ of $(G, X, H)$ such that $C=\tilde{\chi}$. We shall suppose that $\chi: X \longrightarrow \mathcal{S}$ is $\nu$-measurable. This hypothesis will be satisfied if $\mathcal{S}$ is a separable $C^{*}$-algebra, see $[2$, Theorem 10.10]. We define

$$
\begin{aligned}
(f \cdot \xi)(x) & =\int_{G} f(s) \rho\left(s^{-1}, x\right)^{1 / 2} \xi\left(s^{-1} x\right) d \ell_{G}(s), \\
(\xi \cdot g)(x) & =\int_{H} \tilde{\chi}(x, t) \xi(x t) g\left(t^{-1}\right) d \ell_{H}(t), \\
\langle\eta \mid \xi\rangle_{\boldsymbol{B}_{c}}(t) & =\int_{X} \eta(x)^{*} \tilde{\chi}(x, t) \xi(x t) d \nu(x),
\end{aligned}
$$

for all $f \in \mathcal{A}_{c}, \xi, \eta \in \mathcal{V}_{c}$ and $g \in \mathcal{B}_{c}$. We denote by $\mathcal{V}^{C}$ the vector space $\mathcal{V}$ equipped with the above operations.

TheOREM 3.1. $\quad \mathcal{V}^{C}$ is a prehermitian right $\mathcal{B}_{c}$-rigged left $\mathcal{A}_{\mathfrak{c}}$-module.

The proof of this theorem will follow from the next lemma.

LemMa 3.2 .

(i) For any $\xi \in \mathcal{V}^{C},\langle\xi \mid \xi\rangle_{\mathcal{B}_{e}}$ is a positive element of $\mathcal{B}$;

(ii) For any $f \in \mathcal{A}_{c}$ and $\xi \in \mathcal{V}$, we have

$$
\langle f \cdot \xi \mid f \cdot \xi\rangle_{B_{c}} \leqslant\|f\|_{\mathcal{A}}^{2}\langle\xi \mid \xi\rangle_{B_{c}} \quad \text { in } \mathcal{B}
$$

(iii) The linear span of $\left\{f \cdot \xi: f \in \mathcal{A}_{c}, \xi \in \mathcal{V}\right\}$ is dense in $\mathcal{V}$ with respect to the norm induced from $\langle\cdot \mid \cdot\rangle_{B_{c}}$. 
Proof: (i) Choose a covariant representation $(\pi, L)$ of the system $(\mathcal{S}, H, \iota)$ such that the integrated form $\pi \times L$ is faithful. Put

$$
\varepsilon(\xi, v)(x)=\int_{H} \pi(\tilde{\chi}(x, t) \xi(x t)) L_{t} v d \ell_{H}(t)
$$

Then we have

$$
\left\langle(\pi \times L)\left(\langle\eta \mid \xi\rangle_{B_{c}}\right) v \mid w\right\rangle=\int_{X / H}\langle\varepsilon(\xi, v)(x) \mid \varepsilon(\eta, w)(x)\rangle d \mu(\dot{x}) .
$$

Therefore $(\pi \times L)\left(\langle\xi \mid \xi\rangle_{B_{c}}\right)$ is a positive operator, and then $\langle\xi \mid \xi\rangle_{B_{c}}$ is a positive element of $\mathcal{B}$.

(ii) Fix a state $\omega$ of $C^{*}(\mathcal{S}, H, \iota)$. Put

$$
\left\langle\eta \mid \eta^{\prime}\right\rangle_{\omega}=\omega\left(\left\langle\eta \mid \eta^{\prime}\right\rangle_{\mathcal{B}_{c}}\right), \quad \forall \eta, \eta^{\prime} \in \mathcal{V}^{C}
$$

Let $N_{\omega}=\left\{\eta \in \mathcal{V}^{C}:\langle\eta \mid \eta\rangle_{\omega}=0\right\}$, and $q_{\omega}: \mathcal{V}^{C} \longrightarrow \mathcal{V}^{C} / N_{\omega}$ the quotient map, and $\mathcal{H}_{\omega}$ the Hilbert space obtained by completing the space $\mathcal{V}^{C} / N_{\omega}$. For each $s \in G$, we define

$$
(s \cdot \eta)(x)=\rho\left(s^{-1}, x\right)^{1 / 2} \eta\left(s^{-1} x\right), \quad \forall \eta \in \mathcal{V}^{C}
$$

Let $W$ be a compact neighbourhood of the identity $e \in G$. Suppose that $\eta \in \mathcal{V}^{C}$ and suppose that $\eta$ vanishes outside a compact subset $K_{\eta}$. Since $H$ acts properly on $X$, the set

$$
K=\left\{t \in H: W K_{\eta} t \cap W K_{\eta} \neq \phi\right\}
$$

is compact. It then follows that

$$
\left\|\langle s \cdot \eta-\eta \mid s \cdot \eta-\eta\rangle_{B_{c}}\right\|_{1} \leqslant\left\|\Delta_{H}^{-1 / 2} \chi_{K}\right\|_{1}\|s \cdot \eta-\eta\|_{2}^{2}, \quad \forall s \in W .
$$

Define

$$
L_{s}\left(q_{\omega}(\eta)\right)=q_{\omega}(s \cdot \eta), \quad \forall \eta \in \mathcal{V}^{C}
$$

We have

$$
\lim _{s \rightarrow e}\left\|L_{s}\left(q_{\omega}(\eta)\right)-q_{\omega}(\eta)\right\|^{2} \leqslant \lim _{s \rightarrow e}\left\|\langle s \cdot \eta-\eta \mid s \cdot \eta-\eta\rangle_{B_{c}}\right\|_{1}=0
$$

Therefore $L$ defines a continuous unitary representation of $G$ in $\mathcal{H}_{\boldsymbol{\omega}}$.

Let $K_{\xi}$ be a compact set such that $\xi$ vanishes outside $K_{\xi}$, and put $P=\operatorname{supp}(f) K_{\xi}$. Since $s \longmapsto f(s) s \cdot \xi$ is a $\ell_{G}$-measurable map from $G$ into $L^{2}(P, \nu ; \mathcal{S})$, it follows that

$$
f \cdot \xi=\int_{G} f(s) s \cdot \xi d \ell_{G}(s) \quad \text { in } \quad L^{2}(P, \nu ; \mathcal{S})
$$


Observe that the restriction of $q_{\omega}$ to $L^{2}(P, \nu ; \mathcal{S})$ is continuous with respect to the $L^{2}$-norm. Therefore we get

$$
q_{\omega}(f \cdot \xi)=\int_{G} f(s) L_{s}\left(q_{\omega}(\xi)\right) d \ell_{G}(s)=L(f) q_{\omega}(\xi)
$$

It then follows that

$$
\begin{aligned}
\omega\left(\langle f \cdot \xi \mid f \cdot \xi\rangle_{B_{c}}\right) & =\left\|q_{\omega}(f \cdot \xi)\right\|^{2} \\
& \leqslant\|f\|_{\mathcal{A}}^{2}\left\|q_{\omega}(\xi)\right\|^{2}=\|f\|_{\mathcal{A}}^{2} \omega\left(\langle\xi \mid \xi\rangle_{B_{c}}\right) .
\end{aligned}
$$

Since this is true for all states of $\mathcal{B}$, we get the desired inequality.

(iii) For each neighbourhood $V$ of $e$, we choose a positive function $f_{V} \in C_{c}(G)$ such that $\operatorname{supp}\left(f_{V}\right) \subset V$ and $\left\|f_{V}\right\|_{1}=1$. Fix a compact neighbourhood $W$ of $e$. Let $\xi \in \mathcal{V}^{C}$. By arguments similar to those in (ii), there is a constant $\kappa$ (depending on $W$ and $\xi$ ) such that

$$
\left\|\left\langle f_{V} \cdot \xi-\xi \mid f_{V} \cdot \xi-\xi\right\rangle_{B_{c}}\right\|_{1} \leqslant \kappa\left\|f_{V} \cdot \xi-\xi\right\|_{2}^{2}, \quad \forall V \subset W .
$$

With similar arguments to those in [2, Proposition 24.47], we can show that $\lim _{V}\left\|f_{V} \cdot \xi-\xi\right\|_{2}^{2}=0$, and hence

$$
\lim _{V}\left\|\left\langle f_{V} \cdot \xi-\xi \mid f_{V} \cdot \xi-\xi\right\rangle_{B_{c}}\right\|_{B}=0
$$

Corollary 3.3. Let $\gamma$ and $C$ be defined as in Remark 2.3. Then the induced representation obtained by Rieffel's process through the bimodule $\mathcal{V}^{C}$ is unitarily equivalent to $\left(U^{C}, \mathcal{H}^{C}\right)$.

\section{REFERENCES}

[1] N. Bourbaki, Intégration (Hermann, Paris, Chapter 1-4(1965), Chapter 5(1967), Chapter 7-8(1963)).

[2] N. Dinculeanu, Integration on locally compact spaces (Noordhoff International Publishing, Leyden, 1974).

[3] S. Kawakami, 'Irreducible representations of non-regular semi-direct product groups', Math. Japon. 26 (1981), 667-693.

[4] G.W. Mackey, The theory of unitary group representations (The University of Chicago press, 1976).

[5] M.A. Rieffel, 'Induced representations of $C^{*}$-algebras', Adv. Math. 13 (1974), 176-257.

[6] V.S. Varadarajan, Geometry of quantum theory (Springer-Verlag, Berlin, Heidelberg, New York, 1985).

School of Mathematics, Physics,

Computing and Electronics

Macquarie University

New South Wales 2109

Australia 